\title{
Location of the activity responsible for the appearance of coronal mass ejections and the sites of their ejection from the solar surface
}

\author{
N.S. Shilova \\ Institute of Terrestrial Magnetism, Ionosphere and Radio Wave Propagation, 142092 Troitsk, Russia \\ e-mail: nshilova@izmiran.troitsk.ru
}

Received May 31, 1999; accepted February 4, 2000

\begin{abstract}
The white-light films of coronal mass ejections for April - May, 1998 (SOHO LASCO C2, C3), retrieved from Internet, are considered. The positions of active regions on visible and back sides of the Sun for each day are studied. It is shown that a region of large flare can be localized at a distance not less than the solar radius from the place where the transient leaves the solar surface. A perturbation is transferred from the flare to this place within a time not exceeding one minute. The ejection of the CME in the field of view of C2, C3 is determined by its distance from the limb and from the projection of the neutral line of the source surface onto the Sun, rather than by the magnitude of the flare. The source surface is, by definition, a sphere where all field lines are radial. It is believed to exist at a distance 2.5 solar radius from the center of the Sun.
\end{abstract}

Key words: coronal mass ejection - flare disappearance of the solar filament

\section{Introduction}

Coronal mass ejections (CMEs) are important for space weather because they cause large geomagnetic storms. The onsets of CMEs have been associated with both flares and filament eruptions. CME-associated shocks have been studied by Berdichevsky et al. (1998). Much attention has been paid to the study of halo- CMEs (Thompson et al. 1998) because this type of CME is very geoeffective. Much is known about peculiarities of the CME image in different wavelengths (Plunkett et al. 1997) and CME plasmoids (Simnett et al. 1997). However many problems concerning CME origin, connection with large-scale and local solar activity and with other eruptive phenomena are unsolved.

CME onsets in very short time intervals at different position angles on the Sun are at the origin of the multiple CME concept (Lyons et al. 1999). This effect evi- dences the CMEs globality. It is necessary to continue the work in this direction. This paper devoted to the study of CMEs addresses the question of what part of the solar atmosphere is responsible for their appearance.

The beginning of May, 1998 was characterized by the appearance of proton flares 29.04.98, 02.05.98, 06.05.98, 09.05.98 in NOAA 8210 region. All were followed by CME and shock waves in the interplanetary space (radio bursts II type). The region was localized in the southern hemisphere (S 11-18), but the position angles of CME did not correspond to this hemisphere. The present paper is devoted to the investigation of the coordinates of the CME ejection into the interplanetary space and the activity corresponding to its appearance. The level of global solar activity, which was quite low in this period, was favorable for the identification of the sources. We studied movies obtained from the data of SOHO LASCO C2, C3 coronographs (in white light) and of $\operatorname{EIT}(\lambda 195 \mathrm{~A})$ (Delaboudiniere et al. 1995) for the period 24.04-21.05.1998 as well as the activity during this period: subflares, flares, disappearance of filaments, bursts of intensity in the region of $1-8$ A (GOES-8). Hundhausen (1997) showed that the bursts of emission in $1-8 \mathrm{~A}$ are more closely connected (by their localization and the time of appearance) with CME than optical flares. As was shown by Dryer et al. (1998), the peaks of emission in $1-8$ A correspond not only to the instants of flares but also to the instants of CME formation on the solar surface. From this point of view, it is interesting to note that the peaks of this emission are not associated with the appearance of a prominence (Srivastava et al. 1997). Probably GOES 8 did not detect this emission because the site of their origin was not visible (it was on the back side of the Sun). Therefore, we shall assume that if a CME was created but the peak of emission in $1-8 \mathrm{~A}$ was absent at the time of its formation (accounting for the fact that our first observations refer to the height $1.5 R_{\odot}$ above the solar surface), then the region of its formation is localized on the back side of the Sun. For each day of detected 
CME, we should consider active regions, as the most probable places for the respective perturbation, not only on the visible hemisphere but also the regions that can be localized on the back side of the Sun on this day. Such regions could be both the ones that were detectable 13.5 days later and the regions (although with a less probability) that were registered 13.5 days before the event. The last ones were taken into consideration only in the case of their high activity.

It should also be taken into account that white-light observations can be conducted only for the CMEs whose angles are within 35 degrees with respect to the sky plane (Hundhausen 1993), and their displacements along position angles as projected on the sky plane are insignificant up to 55 degrees latitude. If the initial trajectory of the CME is located in the plane perpendicular to the solar surface, then such a CME should be efficiently observed from the active regions localized not farther than 40 degrees from the limb. Let us introduce now such a position of the ejection plane as an assumption, and let the zone within 40 degrees from the limb be called the favorable one.

\section{Analysis of daily observations}

The first day, April 24, is characterized by the absence of sunspots on the visible disk. Nevertheless, an activity of NOAA region 8210 (which has a small area but was located on the east limb) could take place. A small CME appeared in the field of view of C2 at 0927 UT and had a velocity of $330 \mathrm{~km} \mathrm{~s}^{-1}$ (under the assumption of uniform motion). The position angle of its appearance on $\mathrm{E}$ limb was close to the position angle of active region 8210 and the respective angle for the system of loop prominences S34 E90. No streamer was present, but a considerable enhancement of intensity in the line 5303 A took place. No peak of X-rays was observed. So, if an active region is localized in the favorable zone, the CME appears in its vicinity. The motion of the CME on some part of the trajectory can be reasonably considered to be uniform.

On the second day, April 25, the region 8210 appeared on the east disk. The region 8214 was not formed yet, and there were no active regions in the northern hemisphere both in subsequent and previous rotations. During this day, subflares were observed in region 8210; and there were three transients (at 1511, 1640, and 1902 UT) in the field of view of $\mathrm{C} 2$. The last of them evidently showed that the motion of the CME was not uniform: at first, its velocity was $210 \mathrm{~km} \mathrm{~s}^{-1}$; then, $160 \mathrm{~km} \mathrm{~s}^{-1}$; and finally it did not rise at all (i.e., either disappeared or went to the back side of the sky plane). The first two transients could be the consequences of the subflares at 1420 and 1540 UT in region 8210. Transient 1511 UT (the transients will be identified by the instants of their appearance in the $\mathrm{C} 2$ field of view) arose near the position angle of this region.
Transient 1640 was located between the position angle of 8210 and the northern hemisphere of this limb. Transient 1902 arose at an angle of 30 degrees to the north of equator on the west limb. Nothing could correspond to this transient other than the burst $1-8$ A from 8210 , although it was localized far from 8210 but in the streamer.

On the third day, April 26, we can observe only a slight increase of brightness in the $\mathrm{C} 2$ field of view in the streamer at the position angle of the future region 8214. Then, a bifurcation of the streamer occurred. This day is interesting only as an illustration of the fact that the passage of a transient can change the streamer. But this is not a typical response of the streamer to the passage of a transient. Considerably more often, a streamer remains unchanged after the passage of transient, as, for example, on April 29, 1998; or the transient goes beyond the streamer, as is seen from the consideration of days 1 and 2 .

On the fourth day, April 27, region 8210 and region 8199 (on the back side near the east limb at the same latitude) were in the favorable zone. The northern hemisphere still contained no active regions. This day was characterized by a large flare $2 \mathrm{~B} / \mathrm{X} 1$, subflares, and bursts of $1-8 \mathrm{~A}$ emission in region 8210 . A transient in the form of an arch joining the latitudes $25 \mathrm{deg}$. of northern and southern hemispheres arose at $0926 \mathrm{UT}$ in the $\mathrm{C} 2$ field of view (Fig. 1).

Since the X1 burst of the event 2B/X1 began at 0855 , it can be reasonably considered to be responsible for the appearance of the CME. The position of one of the footpoints of the observed CME corresponds to region 8210, but the brightest second footpoint does not have an associated region and is characterized only by a streamer. This is a second case when a CME generated by the event in one active region appears far from this region, in the opposite hemisphere with respect to the equator.

There were no appreciable transients on the fifth day, April 28. On the sixth day, April 29, two events took place: formation of the region 8214, N28 E64, and the flare 3B/M6.8. Of course, there was no activity in the recently formed region 8214. Active region 8210, apart from a large flare, included many bursts of $1-8 \mathrm{~A}$ emission, subflares, and flares IF. CME 1658 appeared and developed only in NE quadrant (Fig. 2).

The speed of its uniform motion can be determined to be $500 \mathrm{~km} \mathrm{~s}^{-1}$. Such an object should leave the surface at $1630 \mathrm{UT}$; this instant coincides with the peak of the burst M6.8 in the event 3B/M6.8. Although our estimates of time are rough, the repetition of situation leads to the conclusion that it is impossible to detect a difference between the instants of CME generation in the vicinity of 8210 and its appearance near 8214. Most probably, this difference is less than 1 minute. As the structure of this CME changed, a concave shape was formed.

The speed of transient motion decreases before the formation of this structure. In this day, apart from the 


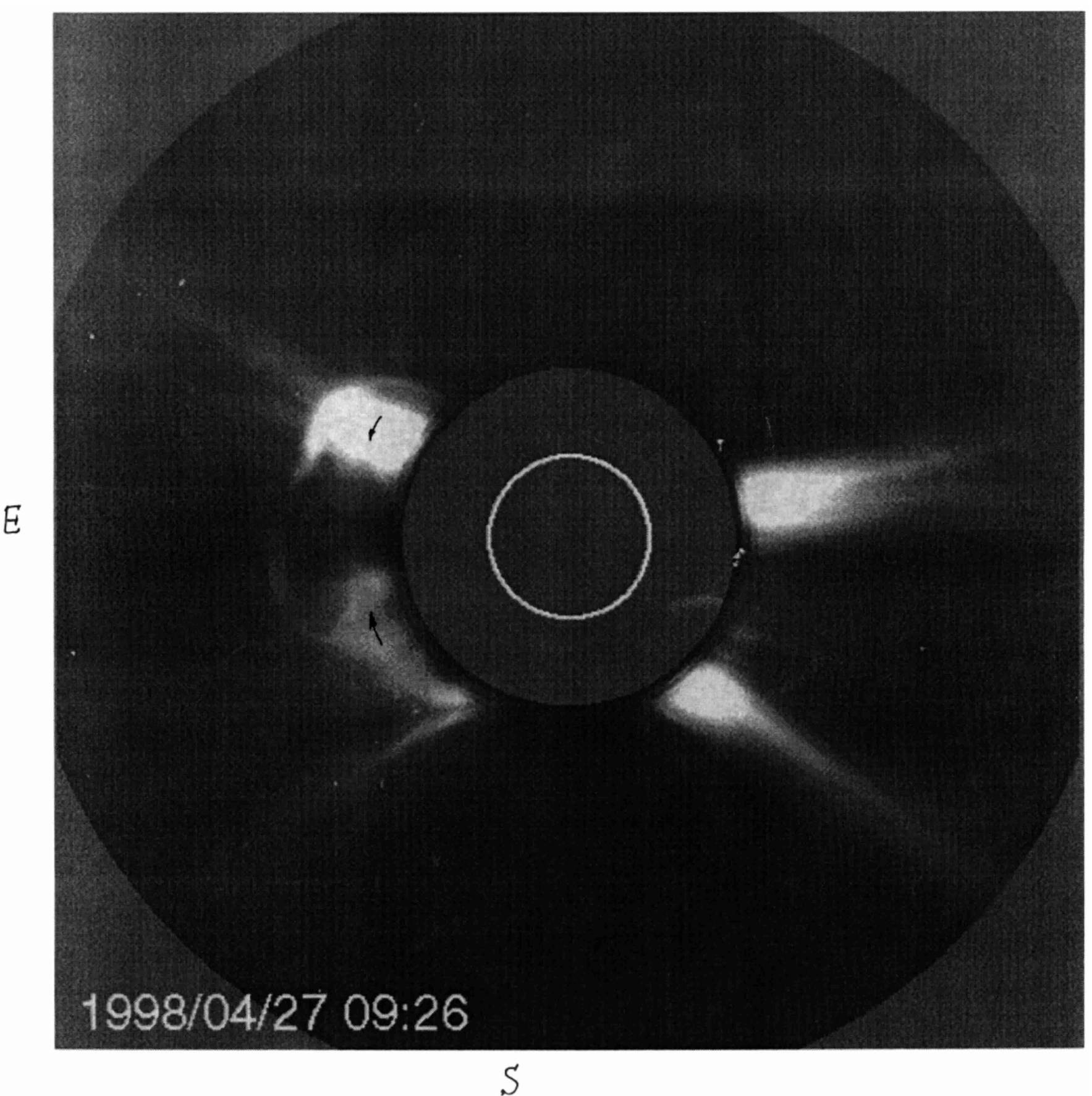

Fig. 1. The CME of April 27, 1998 as observed in white light by the LASCO C2 coronagraph, 0926 UT

transient 1658, a compact plasmoid, moving with a speed $300 \mathrm{~km} \mathrm{~s}^{-1}$, was ejected at 1931 in NW quadrant. Its formation was caused by the activity of region 8222 on the back side of the disk.

During the 3B flare on this day, GOES- 8 detected a long duration event (LDE) of quite complicated form with several maxima (Fig. 3).

The main maximum corresponds to the optical flare; the next maximum at 1720 , to the change in shape of the transient 1658 ; the maximum at 1845 , to the appearance of plasmoid above the solar limb; the maximum at 2030, to the formation of a concave structure in the transient 1658. Consideration of this day and other days in our sequence, for example, May 6, shows that the complex form of LDE is formed not only by flares and subflares but also by the generation of $1-8 \mathrm{~A}$ emission during essential reconstruction of structure of the transient that has left the Sun.

On the seventh day, April 30, region 8210 was far from the favorable zone, near the central meridian; region 8214 


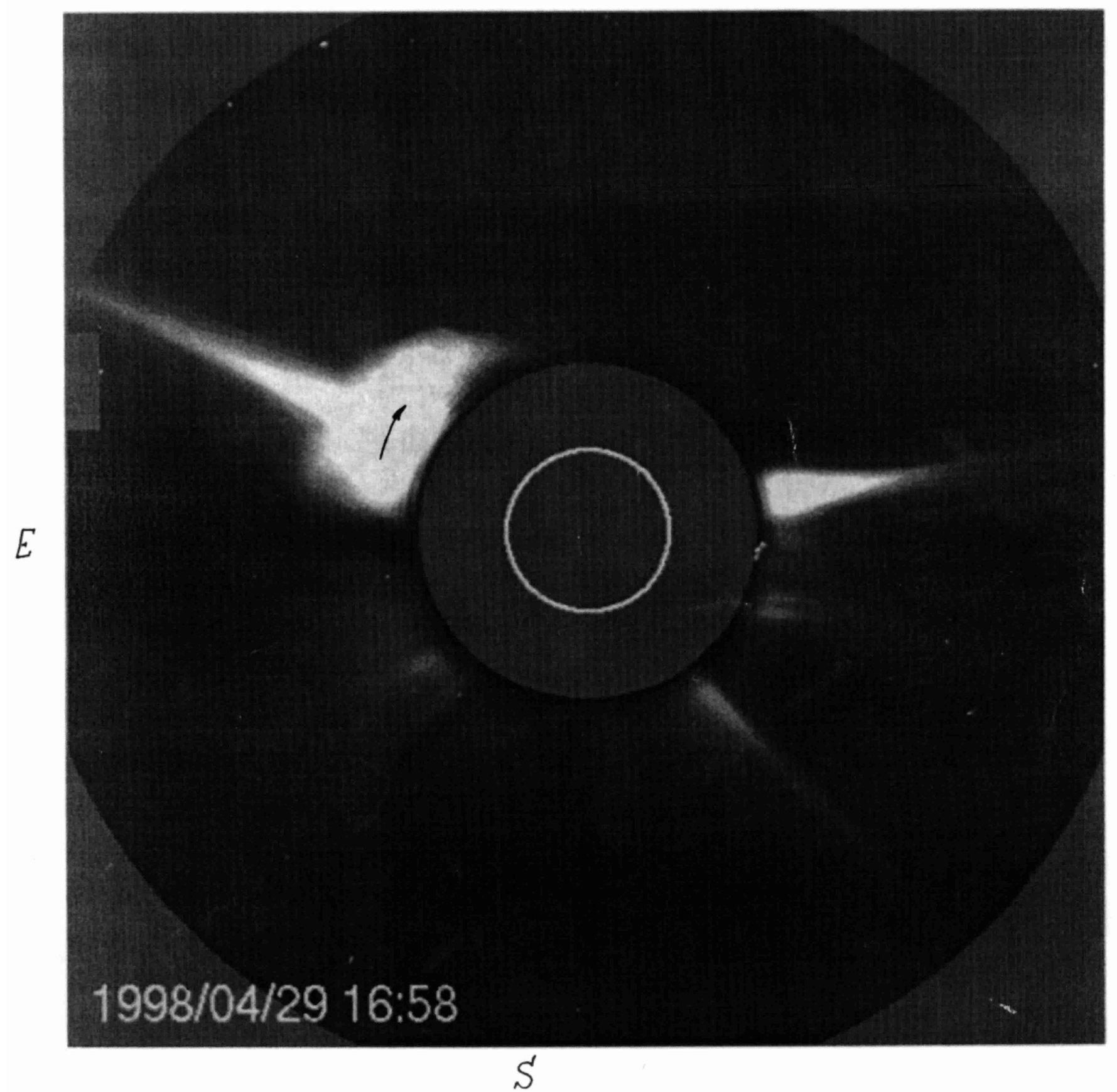

Fig. 2. The CME of April 29, 1998 as observed in white light by the LASCO C2 coronagraph, 1658 UT

was at the boundary of the favorable zone; regions 8217 , 8220,8222 could be in the favorable zone on the back side. Region 8214 produced only subflares; and region 8210 , apart from subflares, produced a $2 \mathrm{~N} / \mathrm{C} 6.6$ event. The appearance of a slight increase of brightness in the $\mathrm{C} 2$ field of view near the west limb can be explained by the action of regions 8220,8222 . Despite an appreciable flare, the east limb was quiet. The last fact confirms the concept of the favorable zone for the observation of transients, since on this day a region with a flare was localized in the unfavorable zone. The absence of $1-8 \mathrm{~A}$ emission bursts at the instants corresponding to the transient confirms the fact that its appearance in this day is associated with activity on the back side.

On the eighth day, May 1 , only regions 8220,8222 (west limb of the back side) and 8217 (east limb of the back side) remained in the favorable zone. Subflares continued to appear in regions 8210,8214 ; and even a 1N/M1.1 flare appeared in 8214. CME 1627 near the west limb in the equatorial zone was caused by the action of groups 8220 and 8222 ; so, it was not detected in X-ray. A flare in 8214 


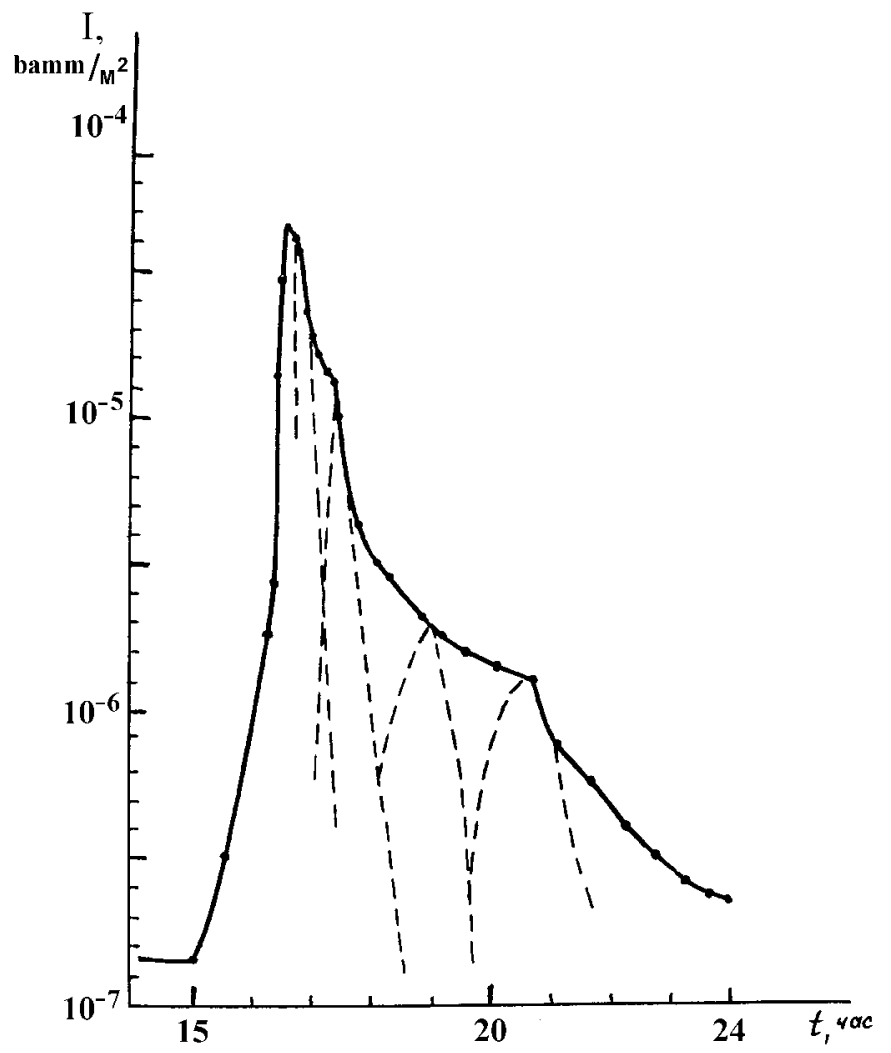

Fig. 3. Long duration event $1-8$ A during 3B/M6.8 flare, April 29, 1998

did not appear in the C2, C3 fields of view, since it was located in the unfavorable zone.

On the ninth day, May 2, only the region 8217 remained in the favorable zone. Nevertheless, there were many flares: a flare IF with eruption, a flare 3B/X1.1 in 8210,9 other subflares in this region, and 6 subflares in region 8214. Transients in this day have small intensity; they can be observed as a "breath" of intensity in a wide range of position angles. Nevertheless, the instants of variation in intensity can be identified with subflares and the flare $3 \mathrm{~B}$ in 8210.

On the tenth day. May 3, we did not have a film for the $\mathrm{C} 2$ and $\mathrm{C} 3$ fields of view.

On the eleventh day, May 4, only region 8218 (back side, S21) was in the favorable zone. Namely this region produced the transient 0627 in the ES quadrant. Region 8210 was in the unfavorable zone and did not exhibit transients, although its activity was up to $1 \mathrm{~F}$ flares.

On the twelfth day, the transients were not observed; but a $2 \mathrm{~N}$ flare appeared at 2329 UT in region 8210 . On this day, region 8210 entered the favorable zone, and the transient corresponding to this flare was observed next day.

May 6. This transient really appeared at 0002 UT near the position angle corresponding to 8210 . There were two other transients in this day. A transient, moving with a velocity of approximately $100 \mathrm{~km} \mathrm{~s}^{-1}$, appeared at $0300 \mathrm{UT}$ in the $\mathrm{C} 2$ field of view in the NE quadrant. It can be explained by the activity of region 8218 on the back side, since there were no manifestations in the range $1-8 \mathrm{~A}$. The third transient 0829 in NW quadrant appeared near region 8214 , its shape changed in a very complicated manner. The speed of its uniform motion at the instant of appearance was about $700 \mathrm{~km} \mathrm{~s}^{-1}$. Consequently, it should have arisen at the solar surface at $0723 \mathrm{UT}$. This time corresponds to lateral X-ray burst of the $1 \mathrm{~N} / \mathrm{X} 2.7$ event in region 8210 . There was no activity at this instant in region 8214 . Therefore, the difference in time between the generation of transient in 8210 and its ejection into the interplanetary space near 8214 in this day also turns out to be undetectable. The localization of the active regions and the trajectory of the transient are such that an assumption on the projection of the CME on the line 8210 - 8214 is not evident. The transient leaves the surface just near 8214. On May 6, the projection of the neutral field line of the source surface onto the Sun passed through this place. The $1 \mathrm{~N} / \mathrm{X} 2.7$ event was exhibited in the band $1-8 \mathrm{~A}$ as a long duration event (LDE), whose complicated form assumed the presence of maxima at 0723 UT (the burst M2.9 in region 8210), at 0815 UT (the burst X2.7 in region 8210), and at 0930 (a reconstruction of interaction between the sides of the CME).

Next day, May 7, the activity of 8214 considerably increased: there were $1 \mathrm{~B}, 1 \mathrm{~F}$ flares, apart from subflares. The transient 0917 showed activity at the position angles between 8210 and 8214 , the velocity of its escape from the surface was $160-190 \mathrm{~km} \mathrm{~s}^{-1}$. The transient 1541, NE, was caused by a subflare and the event C3.7 at 1446 in region 8218 , which was already on the visible side. This transient exhibits the formation of a concave structure, the speed of its motion is about $300 \mathrm{~km} \mathrm{~s}^{-1}$.

On May 8, the CME activity indicates the continuing participation of both 8210 and 8214 in its generation at the position angles of both regions and between them. Successive determination of the velocity shows that the assumption of uniform motion fails, especially, for the C3 field of view. For example, the velocity decreases from $350 \mathrm{~km} \mathrm{~s}^{-1}$ to $150 \mathrm{~km} \mathrm{~s}^{-1}$ before the formation of a plasmoid.

On May 9, regions 8214 (west limb) and 8218 (east limb) were in the favorable zone on visible side; region 8210 was in the favorable zone on the back side near the west limb. At 0335 the transient 0235 took the form of an arch, joining regions 8210 and 8214. A large flare in 8210 on the back side of the limb (event M7.7) is responsible for this arch. Region 8214 was located at the position angle corresponding to a streamer. The west limb repeatedly generated transients throughout this day. We can see some similarity of these transients with transients which occurred on May 8: arches crossing the equator, their evolution followed by the appearance of an arch at the neighboring position angle, the increase in brightness of one leg during the disappearance of arches. 

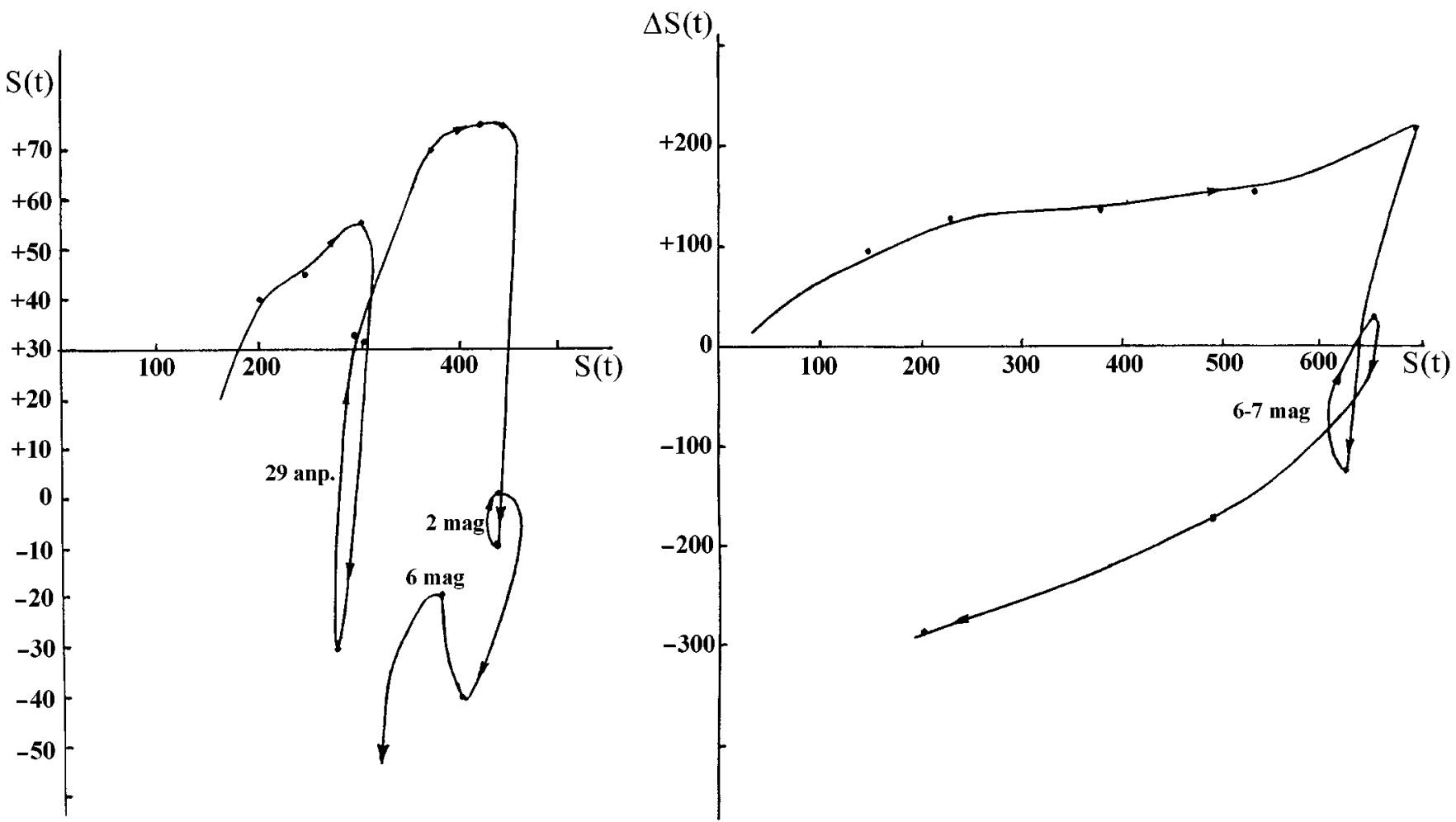

Fig. 4. Phase diagrams of active regions 8210 and 8214. $S(t)$ - the daily total area of sunspots in millionth parts of hemisphere, $\delta S(t)$ - the variaty of these values

On May 10, transients 1527 and 0000 in the SW quadrant are associated with the disappearance of a filament and a system of loop prominences at the position angle corresponding to region 8210. As follows from the area of sunspots, region 8214 almost disappeared.

On subsequent days, region 8210 went away from the favorable zone. The observed transients were caused by the action of small regions 8218,8214 , and, probably, 8217 , as well as by the disappearance of filaments.

On May 19, 20, region 8210 should enter the favorable zone near the east limb on the back side. But since May 20 , this region did not reveal itself in the generation of CME. One can assume identify of this region with region 8225 which appeared on May 22 at latitude SI8. But its area was negligibly small (only 10 millionth parts of the visible hemisphere), in subsequent time it did not exceed 20 millionth parts; and this region could hardly be the source of a CME.

Let us consider the question if regions 8214 and 8210 refer to the same complex of activity. None of the signs pointing to the relation of these solar regions to the same complex of activity (Mogilevskij \& Shilova 1998) is satisfied here. Ha fibrils do not join these regions in space, the regions exist by themselves (Big Bear filtergrams). Largescale magnetic fields of Carrington revolution 1935 from April 24 to May 10 produce structures that do not go from the southern to northern hemisphere. The curves of variation of the total area of sunspots in the regions (in the units of their maximal values) considerably differ from each other and do not reveal similar features. The phase pictures of regions $S(t)-\delta S(t)$ are also dissimilar. For example, a phase diagram of 8210 has loops on April 29, May 2-3, and May 6- the days of maximal flare activity. On the other hand, the phase diagram of 8214 has a loop only on May 6-7 (Fig. 4).

Therefore, regions 8214 and 8210 do not refer to the same complex of activity; and, consequently, their coupling below the photosphere should not be strong. Nevertheless, the loops on the Yohkoh filtergram show an arcade joining these regions. The respective $\mathrm{X}$-ray bridge was seen very clearly on May 6, against the background of a coronal hole. As is seen in the films for $195 \mathrm{~A}$ band, these regions are < breathing $>$ in synchrony; and bright points simultaneously appear in both regions. It should be mentioned that from April 29 to May 7 region 8214 was near the projection of the neutral field line of the source surface onto the Sun; but region 8210 was far from this line.

\section{Conclusion}

Our investigation showed that the coupling between the regions in several cases was so efficient that at the instant of a flare in one of these regions the transient was detected near the other region. While the regions were on the east edge of the visible disk, there was no streamer near the 
region with a flare, and in the respective quadrant at all. In other words, the neutral field line of the source surface did not go through the position angle of this active region. When the region was near the west limb, the streamer either went very close to the pole (May 4,6) or was absent in this quadrant (May 9). The neutral line of a global magnetic field is probably associated with the place where the transients usually leave the surface. It seems most likely that the quick transmission of influence from 8210 to 8214 is caused by electric currents of the magnetic loops. This problem should be studied in more detail. Our preliminary analysis leads to the conclusion that there are two mechanisms for the formation of transients detected in the C2, C3 field of view. The first mechanism: a spiral ejection with high velocities is generated in the active region; a transient, going into the interplanetary space, appears in the same place; the instability, producing the ejection, is transmitted approximately within one hour below the photosphere to the associated region in this complex of activity, where a transient is formed in a similar way; then, these transients interact with each other in the field of view of C3 (Mogilevskij et al. 1999). The second mechanism: the ejection appears at the site of an instability (i.e., a flare); the excitation is very quickly transmitted through the coronal loops to the place on the solar surface where the escape of transient is the easiest. Under favorable location of the region of generation, it simultaneously produces another transient. As a result, we get the concepts of the multiple CME (Dere et al. 1998; Lyons et al. 1998).

Therefore, our consideration of a sequence of C2, C3 movies describing the motion of regions with flares over the disk, shows that:

1) a CME can not only appear near the region of the generating instability but also near the site located at a distance of the order of one solar radius from that region. In this case, the influence is transmitted very quickly. The proximity of projection of the neutral line of the source surface field is favorable for the escape of transient;

2) interaction of structural objects of a transient can be followed by the generation of $1-8 \mathrm{~A}$ emission, whose instants coincide with maxima instants of the long duration burst in 1-8 A, commonly observed in considerable flares.

In addition it is shown that under favorable conditions for the escape from the surface, subflares may generate CME. The hypothesis of observation of intensive CME only from the regions localized within 40 degrees from the solar limb is confirmed. The presence (or absence) of burst in the range $1-8 \mathrm{~A}$ at the instant predicted for the appearance of the transient near the surface is the evidence that an event on the visible (or back) side of the Sun is responsible for the generation of the CME.

Acknowledgements. The author is very grateful to Dr. R. Howard for the possibility of using the C2, C3, 195 A movies via Internet.

This work was carried out by the financial support of the Russian Foundation for Basic Research (grants Nos. 96-02-16285, 96-02-16827a) and the Astronomy State Program.

\section{References}

Berdichevsky D., Bougeret J.-L., Delaboudiniere J.-P., et al., 1998, Geophys. Res. Lett. 25, 2473

Delaboudiniere J.-P., Artzner G.E., Brunaud J., et al., 1995, Sol. Phys. 162, 291

Dere K.P., Howard R.A., Brueckner G.E., LASCO and EIT observations, 1998, Am. Geophys. Un. spring meeting, Boston, Massachusetts, Suppl. to EOS Trans., p. 257

Dryer M., Andrews M.D., Aurass H., 1998, Sol. Phys. 181, 159 Hundhausen A.J., 1993, J. Geophys. Res. 98, 13177

Hundhausen A.J., 1997, Coronal mass ejections, in Jokipii J.R. et al. (eds.), Cosmic winds and heliosphere. Univer. of Arizona Press, Tuscon, p. 259

Lyons M.A., Simnett G.M., St.Cyr O.C., Thompson B., 1998, A multiple CME event observed with LASCO on 2-3 June 1997, Am. Geophys. Un. spring meeting. Boston, Massachusetts, Suppl. to EOS Trans., p. 258

Lyons M.A., Simnett G.M., 1999, Sol. Phys. 186, p. 363

Mogilevskij E.I., Shilova N.S., 1998, Izv. Krymskoi Astr. Obs. 94,60

Mogilevskij E.I., Obridko V.N., Shilova N.S., 1999, Soviet Astron. 76, 299

Plunkett S.P., Brueckner G.E., Dere K.P., et al., 1997, Sol. Phys. 175,699

Simnett G.M., Jappin S.J., Plunkett S.P., et al., 1997, Sol. Phys. 175, 685

Srivastava N., Gonzalez W.D., Gonsalez A.L.C., Masuda S., 1997, on the characteristics of solar origin of geoeffective CMEs observed during August 1992-April 1993, in Wilson A. (ed.), Correlated phenomena at the Sun, in the heliosphere and in geospace, The 31 ESLAB Symp. Noordwijk, Netherlands, p. 443

St.Cyr O.C., Howard R.A., Simnett G.M., et al., 1997, White-light coronal mass ejections: A new perspective from LASCO, Ibid, p. 103

Thompson B.J., Plunkett S.P., Gurman G.B., et al., 1998, Geophys. Res. Lett. 25, 2465 